\title{
JOINT STATE AND MEASUREMENT TIME-DELAY ESTIMATION OF NONLINEAR STATE SPACE SYSTEMS
}

\author{
- INVITED PAPER - \\ John-Olof Nilsson ${ }^{\diamond}$ Isaac Skog, and Peter Händel \\ Signal Processing Lab \\ ACCESS Linnaeus Centre \\ Royal Institute of Technology (KTH) \\ SE-100 44, Stockholm, Sweden \\ E-mail: `jnil02@kth.se
}

\begin{abstract}
Sensor fusion algorithms often assume perfect time synchronization of the sensor clocks. In a practical sensoractuator setup this is often difficult to achieve which in turn can give rise to systematic errors in the sensor fusion. In this article we suggest how the effect of the synchronization error from an unknown static or slowly varying measurement time-delays in a nonlinear state space system can be handled by linearizing the measurement equation in time. Based on the linearization an augmented system is constructed from which the system states and the delays can be jointly estimated. Expressions for the system, measurement, and covariance matrices of the augmented system are derived. Finally, the feasibility of the suggested approach is demonstrated by an example and a Monte-Carlo simulation.
\end{abstract}

Index Terms - Delay estimation, state estimation, synchronization, nonlinear systems

\section{INTRODUCTION}

Common sensor fusion algorithms such as Kalman filter based methods normally assume perfect time synchronization in between different measurements and between measurements and input signals. In reality this is seldom the case. In a practical sensor-actuator setup there will inevitably be time uncertainties in the sampling instances, whose effects on the sensor fusion are often not straightforward how to handle. Ignoring these uncertainties will in general lead to systematic errors in the sensor fusion. The time uncertainties can be both positive and negative depending on the time reference. However, they are commonly referred to as delays which is why we will stay with that terminology.
Treatments of unknown delays found in the literature can be divided into two cases. First, the delays are unknown and random with a short (zero) correlation time (time jitter), see for example [1,2]. Second, the delays are unknown but static or varying slowly. The latter is the case studied in this article. The case can be motivated by the result that through filtering of the time stamps from time-driven sensors one can achieve a static or slowly varying synchronization error independent of the measurement content [3]. Traditionally the unknown static delays have been treated with system identification methods $[4,5]$. However, these methods are often limited to linear systems. In a recent approach suggested in [6] the problem is solved, for a nonlinear state space system, by appending the unknown delay and a noise state to the system state, and by evaluating the measurement function at an intermediate time instance by propagating the states through the nonlinear system function. This way a new measurement function is achieved and the delay can be estimated jointly with the system states. Similarly, in [7], for the special case of GPS-aided inertial navigation, the delay is jointly estimated by making a second order Taylor serie approximation of the measurement model with respect to the delay and feeding the estimated delay back to a fractional delay filter acting on the incoming measurements.

In this article we suggest a novel approach to the measurement delay estimation problem of the nonlinear state space system. The effect of the unknown delays of an arbitrary number of measurement sources are evaluated by linearizing the measurement equations in time. An augmented system is constructed within which the delays can be estimated jointly, together with the original states, by an extended Kalman filter (EKF). Expressions for the system, measurement, and covariance matrices are derived and a nonlinear example is worked out and simulated to show the feasibility of the approach. 


\section{SYSTEM DESCRIPTION}

The plant of interest is assumed to be modeled by a continuous nonlinear system described by a first order differential equation system,

$$
\dot{\mathbf{x}}(t)=f_{c}(\mathbf{x}(t), \mathbf{u}(t))+\mathbf{w}(t),
$$

where $\mathbf{x}(t) \in \mathbb{R}^{N}$ denotes the system states, $\mathbf{u}(t)$ the system input, and $\mathbf{w}(t)$ a process noise. To the plant there are sensors giving discrete measurements,

$$
\mathbf{y}_{i}(k)=h_{i}(\mathbf{x}(k))+\mathbf{v}_{i}(k) \quad i=1,2, \ldots
$$

where $i$ is a sensor index and $k \in \mathbb{N}$ is a time index of the sampling instances $t_{k}$ of the sensors ( $k$ is taken as short notation for $t_{k}$ ).

For implementability on digital devices the system (1) is assumed to have been discretized,

$$
\mathbf{x}(k+1)=f_{k}(\mathbf{x}(k), \mathbf{u}(k))+\mathbf{w}(k),
$$

where $\mathbf{w}(k)$ is the sampled process noise (assumed resulting in a white noise) and where $f_{k}(\cdot)$ has a $k$-dependence since $k$ does not necessary correspond to even sampling periods. Even though (3) is given, the implicit assumption of an underlying continuous system (1) is crucial, since, otherwise, the delay would not be continuous and the delay estimation would be a detection problem.

Note that the description (3) includes solution of (1) by numerical methods as well as simple zero-order-hold approximations. The only requirement we put on (3) is that it gives means of propagating the system state from time instances $t_{k}$ to time instance $t_{k+1}$. However, later in equations (11), (12), and (19) we restrict the discretization to zero-order-hold type since it gives simple propagations of the filter equations. Alternatively, if for example RungeKutta-like approximations are used in (3) then the same approximations ought to be used instead of (19).

Unfortunately, since, apart from $\mathbf{y}_{i}(k), \mathbf{u}(t)$ is also typically a measured quantity, how to achieve (3) for all $k$ is not straight-forward. However, in practice $\mathbf{u}(t)$ is often measured at a higher rate than the other measurements, an internal signal available for chosen instances, or a quantity that we can interpolate treating the interpolation error as a white noise. Hence, we will assume that $\mathbf{u}(t)$ is given for all $k$ and all $t$ needed for the update in (3).

\section{DELAYED MEASUREMENTS}

In a practical sensor-actuator setup there will often be unknown delays in the measurements. Taking the time of the input $\mathbf{u}(t)$ to be the reference time, the delays will shift the measurement models (2) in time such that

$$
\mathbf{y}_{i}(k)=h_{i}\left(\mathbf{x}\left(k+\delta t_{i}\right)\right)+\mathbf{v}_{i}(k)
$$

where $\delta t_{i}$ are the unknown delays. For small delays this can be evaluated by making Taylor serie expansions of $h_{i}(\cdot)$ in time around the nominal measurement time instances $k$,

$$
\begin{aligned}
\mathbf{y}_{i}(k)= & h_{i}\left(\mathbf{x}\left(k+\delta t_{i}\right)\right)+\mathbf{v}_{i}(k) \\
= & h_{i}(\mathbf{x}(k))+\left.\frac{\partial h_{i}(\mathbf{x}(t))}{\partial t}\right|_{k}\left(\left(k+\delta t_{i}\right)-k\right) \\
& +\ldots+\mathbf{v}_{i}(k)
\end{aligned}
$$

Discarding higher order terms and by the chain rule,

$$
\begin{aligned}
\mathbf{y}_{i}(k) & \simeq h_{i}(\mathbf{x}(k))+\left.\frac{\partial h_{i}(\mathbf{x}(t))}{\partial t}\right|_{k} \delta t_{i}+\mathbf{v}_{i}(k) \\
& =h_{i}(\mathbf{x}(k))+\left.\frac{\partial h_{i}(\mathbf{x}(t))}{\partial \mathbf{x}(t)} \frac{\partial \mathbf{x}(t)}{\partial t}\right|_{k} \delta t_{i}+\mathbf{v}_{i}(k)
\end{aligned}
$$

where $\simeq$ denotes a quantity where only the dominant terms are retained. Defining the measurement matrix

$$
\mathbf{H}_{i}(\mathbf{x}(k))=\left.\frac{\partial h_{i}(\mathbf{x})}{\partial \mathbf{x}}\right|_{\mathbf{x}(k)}
$$

and using (1), (5) can be written as

$$
\begin{aligned}
\mathbf{y}_{i}(k)= & h_{i}(\mathbf{x}(k))+\mathbf{H}_{i}(\mathbf{x}(k)) f_{c}(\mathbf{x}(k), \mathbf{u}(k)) \delta t_{i} \\
& +\mathbf{H}_{i}(\mathbf{x}(k)) \delta t_{i} \mathbf{w}(k)+\mathbf{v}_{i}(k) .
\end{aligned}
$$

\section{AUGMENTED SYSTEM}

To compensate for the effect of the delays we want to estimate them jointly together with the original states. For this an augmented system with the state vector from (3) appended with the delays $\delta t_{i}$ is constructed,

$$
\begin{aligned}
& \mathbf{x}^{\prime}(k+1)=f_{k}^{\prime}\left(\mathbf{x}^{\prime}(k), \mathbf{u}(k)\right)+\mathbf{w}^{\prime}(k) \\
&=\left[\begin{array}{c}
f_{k}(\mathbf{x}(k), \mathbf{u}(k)) \\
\delta t_{1} \\
\vdots \\
\delta t_{M}
\end{array}\right]+\mathbf{w}^{\prime}(k), \\
& \text { where } \quad \mathbf{x}^{\prime}(k)=\left[\begin{array}{c}
\mathbf{x}(k) \\
\delta t_{1} \\
\vdots \\
\delta t_{M}
\end{array}\right] \quad \text { and } \quad \mathbf{w}^{\prime}(k)=\left[\begin{array}{c}
\mathbf{w}(k) \\
\mathbf{0}_{M \times 1}
\end{array}\right],
\end{aligned}
$$

and where $M$ denotes the number of delays modeled. If the delays are varying slowly, to model the drift, a driving noise can be added to the $M$ last dimension of $\mathbf{w}^{\prime}(k)$. This would necessarily also add a time index to $\delta t_{i}$. The measurement models of the delayed measurements are taken from (7) as

$$
\begin{aligned}
\mathbf{y}_{i}(k)= & h_{i}^{\prime}\left(\mathbf{x}^{\prime}(k), \mathbf{u}(k)\right)+\mathbf{v}_{i}^{\prime}(k) \\
= & h_{i}(\mathbf{x}(k))+\mathbf{H}_{i}(\mathbf{x}(k)) f_{c}(\mathbf{x}(k), \mathbf{u}(k)) \delta t_{i} \\
& +\mathbf{v}_{i}^{\prime}(k)
\end{aligned}
$$

where the new measurement noise vectors are

$$
\mathbf{v}_{i}^{\prime}(k)=\mathbf{H}_{i}(\mathbf{x}(k)) \delta t_{i} \mathbf{w}(k)+\mathbf{v}_{i}(k) .
$$




\section{STATE AND DELAY ESTIMATION}

The augmented state space system, (8) and (9), can be estimated by an EKF. In this case we will have a first order approximation (linearization) of a first order approximation with respect to the delay. However, this still gives a first order approximation.

The system matrix of the original system is defined by

$$
\mathbf{F}_{k}(\mathbf{x}(k), \mathbf{u}(k))=\left.\frac{\partial f_{k}(\mathbf{x}, \mathbf{u})}{\partial \mathbf{x}}\right|_{\mathbf{x}(k), \mathbf{u}(k)} .
$$

Together with (8) this gives the system matrix of the augmented system

$$
\begin{aligned}
\mathbf{F}_{k}^{\prime}\left(\mathbf{x}^{\prime}(k), \mathbf{u}(k)\right) & =\left.\frac{\partial f_{k}^{\prime}\left(\mathbf{x}^{\prime}, \mathbf{u}\right)}{\partial \mathbf{x}^{\prime}}\right|_{\mathbf{x}^{\prime}(k), \mathbf{u}(k)} \\
& =\left[\begin{array}{cc}
\mathbf{F}_{k}(\mathbf{x}(k), \mathbf{u}(k)) & \mathbf{0}_{N \times M} \\
\mathbf{0}_{M \times N} & \mathbf{I}_{M \times M}
\end{array}\right] .
\end{aligned}
$$

whereas straight-forward calculations give the measurement matrices of the augmented system

$$
\begin{aligned}
& \mathbf{H}_{i}^{\prime}\left(\mathbf{x}^{\prime}(k), \mathbf{u}(k)\right)=\left.\frac{\partial h_{i}^{\prime}\left(\mathbf{x}^{\prime}, \mathbf{u}\right)}{\partial \mathbf{x}^{\prime}}\right|_{\mathbf{x}^{\prime}(k), \mathbf{u}(k)} \\
& =\left[\mathbf{H}_{i}(\mathbf{x}(k))+\mathbf{D}_{i}(\mathbf{x}(k), \mathbf{u}(k)) \delta t_{i} \quad \mathbf{0}_{\operatorname{dim}\left(\mathbf{y}_{i}\right) \times i-1}\right. \\
& \left.\mathbf{H}_{i}(\mathbf{x}(k)) f_{c}(\mathbf{x}(k), \mathbf{u}(k)) \quad \mathbf{0}_{\operatorname{dim}\left(\mathbf{y}_{i}\right) \times M-i}\right]
\end{aligned}
$$

where $\mathbf{D}_{i}(\mathbf{x}(k), \mathbf{u}(k))$ is a matrix whose $j$ :th row (superscript $(j))$ is

$$
\begin{aligned}
\mathbf{D}_{i}^{(j)}(\mathbf{x}(k), \mathbf{u}(k))= & \left.f_{c}(\mathbf{x}(k), \mathbf{u}(k))^{T} \frac{\partial^{2} h_{i}^{(j)}(\mathbf{x})}{\partial \mathbf{x}^{2}}\right|_{\mathbf{x}(k)} \\
& +\left.\mathbf{H}_{i}^{(j)}(\mathbf{x}(k)) \frac{\partial f_{c}(\mathbf{x}, \mathbf{u})}{\partial \mathbf{x}}\right|_{\mathbf{x}(k), \mathbf{u}(k)}
\end{aligned}
$$

and where $\frac{\partial^{2} h_{i}^{(j)}(\mathbf{x})}{\partial \mathbf{x}^{2}}$ is the Hessian of the $j$ :th dimension (row) of $h_{i}(\mathbf{x})$.

The additional quantities needed for the EKF are the process noise and the measurement noise covariances. The covariances matrices of the original system are assumed give,

$$
\begin{aligned}
\mathbf{Q}(k) & =\operatorname{cov}(\mathbf{w}(k), \mathbf{w}(k)), \\
\mathbf{R}_{i}(k) & =\operatorname{cov}\left(\mathbf{v}_{i}(k), \mathbf{v}_{i}(k)\right),
\end{aligned}
$$

and the cross covariance

$$
\mathbf{S}_{i}(k)=\operatorname{cov}\left(\mathbf{w}(k), \mathbf{v}_{i}(k)\right)=\mathbf{0}_{\operatorname{dim}\left(\mathbf{y}_{i}\right) \times N} .
$$

Together with (8), (9), and (10) they give the covariances matrices of the augmented system,

$$
\begin{aligned}
\mathbf{Q}^{\prime}(k) & =\operatorname{cov}\left(\mathbf{w}^{\prime}(k), \mathbf{w}^{\prime}(k)\right) \\
& =\left[\begin{array}{cc}
\mathbf{Q}(k) & \mathbf{0}_{N \times M} \\
\mathbf{0}_{M \times N} & \mathbf{0}_{M \times M}
\end{array}\right], \\
\mathbf{R}_{i}^{\prime}(k) & =\operatorname{cov}\left(\mathbf{v}_{i}^{\prime}(k), \mathbf{v}_{i}^{\prime}(k)\right) \\
& =\mathbf{H}_{i}(\mathbf{x}(k)) \mathbf{Q}(k) \mathbf{H}_{i}^{T}(\mathbf{x}(k)) \delta t_{i}^{2}+\mathbf{R}_{i}(k),
\end{aligned}
$$

and

$$
\begin{aligned}
\mathbf{S}_{i}^{\prime}(k) & =\operatorname{cov}\left(\mathbf{w}^{\prime}(k), \mathbf{v}_{i}^{\prime}(k)\right) \\
& =\left[\begin{array}{c}
\mathbf{Q}(k) \mathbf{H}_{i}^{T}(\mathbf{x}(k)) \delta t_{i} \\
\mathbf{0}_{M \times \operatorname{dim}\left(\mathbf{y}_{i}\right)}
\end{array}\right] .
\end{aligned}
$$

Based on equations (8)-(18) the well known Kalman filter equations of the predict phase are [8]

$$
\begin{aligned}
\hat{\mathbf{x}}^{\prime}-(k)= & f_{k}^{\prime}\left(\hat{\mathbf{x}}^{\prime}+(k-1), \mathbf{u}(k)\right) \\
\mathbf{P}_{k}^{-}= & \hat{\mathbf{F}}_{k-1}^{\prime} \mathbf{P}_{k-1}^{+} \hat{\mathbf{F}}_{k-1}^{\prime T}+\mathbf{Q}_{k}^{\prime} \\
& -\hat{\mathbf{S}}_{i, k-1}^{\prime}\left(\hat{\mathbf{R}}_{i, k-1}^{\prime}+\hat{\mathbf{H}}_{i, k-1}^{\prime}\right) \hat{\mathbf{S}}_{i, k-1}^{\prime T} \\
& -\hat{\mathbf{F}}_{k-1}^{\prime} \mathbf{K}_{i, k-1} \hat{\mathbf{S}}_{i, k-1}^{\prime T} \\
& -\hat{\mathbf{S}}_{i, k-1}^{\prime} \mathbf{K}_{i, k-1}^{T} \hat{\mathbf{F}}_{k-1}^{\prime T}
\end{aligned}
$$

and of the update phase

$$
\begin{aligned}
\mathbf{K}_{i, k}= & \left(\mathbf{P}_{k}^{-} \hat{\mathbf{H}}_{i, k}^{\prime T}+\hat{\mathbf{S}}_{i, k}\right) \\
& \times\left(\hat{\mathbf{H}}_{i, k}^{\prime} \mathbf{P}_{k}^{-} \hat{\mathbf{H}}_{i, k}^{\prime T}+\hat{\mathbf{R}}_{k}^{\prime}\right)^{-1} \\
\hat{\mathbf{x}}^{\prime}+(k)= & \hat{\mathbf{x}}^{\prime}-(k)+\mathbf{K}_{i, k}\left(\mathbf{y}_{i}(k)-h_{i}^{\prime}\left(\hat{\mathbf{x}}^{\prime}-(k), \mathbf{u}(k)\right)\right) \\
\mathbf{P}_{k}^{+}= & \mathbf{P}_{k}^{-}-\mathbf{K}_{i, k} \hat{\mathbf{H}}_{i, k}^{\prime} \mathbf{P}_{k}^{-}
\end{aligned}
$$

where, for clarity and compactness, the dependence on the $\mathbf{x}^{\prime}(k), \mathbf{u}(k)$, and the time $k$ has been implicitly expressed though a subscript $k$ and where ${ }^{\wedge}$ denotes an estimated value or a matrix evaluated at an estimated value. $\mathbf{P}_{k}$ is the estimate error covariance and ${ }^{-}$and ${ }^{+}$denote the predicted and updated version of an estimated quantity. $\hat{\mathbf{x}}^{\prime}-(k)=\hat{\mathbf{x}}^{\prime}(k), \hat{\mathbf{P}}_{k}^{-}=\hat{\mathbf{P}}_{k}^{+}$, and $\hat{\mathbf{S}}_{i, k}=\mathbf{0}$ if there is no measurement (update possibility) at instance $k$.

Note that the formulas are significantly simplified if $\hat{\delta} t_{i}^{+}=0, \mathbf{Q}_{k} \hat{\mathbf{H}}_{i, k}^{T}=\mathbf{0}$, or if $\mathbf{w}_{k}=\mathbf{0}_{N \times 1}$, which independently of each other give $\hat{\mathbf{S}}_{i, k}=\mathbf{0} . \hat{\delta} t_{i}^{+}=0$ can be achieved by feeding the estimated delay back to the time stamping of the measurements (assignment of $k$ ). That is changing the linearization point of (4), see [7] for details. Modifying the time stamps $k$ of the measurements by the estimated delay sets the estimated delay to zero. In contrast to $\mathbf{Q}_{k} \hat{\mathbf{H}}_{i, k}^{T}=\mathbf{0}$ and $\mathbf{w}_{k}=\mathbf{0}_{N \times 1}$ this also ensures that the linearization (4) is done at the best point given the delay estimate $\hat{\delta} t_{i}^{+}$. An alternative way would be to feed back only a part of the delay corresponding to an even sampling period. This way only a shift in the order of sampling instances of different sensors has to be done while still achieving a good linearization point and keeping the cross covariance $\hat{\mathbf{S}}_{i, k}$ small.

\section{EXAMPLE AND SIMULATION}

We here illustrate the presented approach to joint state and measurement time-delay estimation by a simulated low dimensional example. For a more advanced example in a similar framework see [7].

Consider a compression pump powered by an electrical engine. The change in rotation speed of the pump is proportional to the power supplied to the engine minus a linear friction term and minus an exponential term describing 
the pressure resistance that the pump is working against. Measuring the over-pressure, in the system supplied by the pump, with a sampling period $d t_{y}$ and the input voltage to the engine with a sampling period $d t\left(d t>d t_{y}\right)$, we are interested in the response (measurement) delay between the input voltage and the pressure. In the example only $\delta t \leq 0$ is physically reasonable. However, for completeness $\delta t>0$ is also shown.

The system dynamic is modeled by

$$
\begin{aligned}
\dot{\theta}(t)= & \omega(t) \\
\dot{\omega}(t)= & -\mu \omega(t)-\nu \exp \left(\eta\left(\omega(t)-\omega_{0}\right)\right)+\frac{J}{R} u^{2}(t) \\
& +w(t)
\end{aligned}
$$

where $\theta(t)$ is the angular orientation of the system, $\omega(t)$ is the angular rate, $u(t)$ is the input voltage, $w(t)$ is a process noise which becomes white with variance $\sigma_{w}^{2}$ when sampled with period $d t$, and $\mu, \nu, \eta, \omega_{0}, J$ and $R$ are system constants. From steady state measurements (steady $\omega)$ the down-stream over-pressure has been determined to be proportional to the exponential term and with a significant frequency component due to the system rotation (angular orientation). The pressure measurements are therefore modeled by

$$
\begin{aligned}
y(k)= & \gamma(\sin (\theta(k+\delta t))-1) \\
& +\rho \exp \left(\eta\left(\omega(k+\delta t)-\omega_{0}\right)\right)+v(k)
\end{aligned}
$$

where $v(k)$ is a white Gaussian measurement noise with variance $\sigma_{v}^{2}, \gamma$ and $\rho$ are system constants, and where $\delta t$ is the response delay of the system.

The system is discretized with a zero-order hold and a first-order matrix exponent approximation,

$$
\begin{aligned}
\theta(k+1)= & \theta(k)+d t \omega(k) \\
\omega(k+1)= & \omega(k)-d t \mu \omega(k)-d t \nu \exp \left(\eta\left(\omega(k)-\omega_{0}\right)\right) \\
& +d t \frac{J}{R} u^{2}(k)+w(k)
\end{aligned}
$$

where $w(k)$ is the white sampled process noise. From (23) and (24) the system matrix (11) and the process noise covariance matrix (14) are

$$
\mathbf{F}(\omega(k))=\left[\begin{array}{cc}
1 & d t \\
0 & 1-d t \mu-d t \nu \exp \left(\eta\left(\omega(k)-\omega_{0}\right)\right)
\end{array}\right]
$$

and

$$
\mathbf{Q}=\left[\begin{array}{cc}
0 & 0 \\
0 & \sigma_{w}^{2}
\end{array}\right]
$$

Further, from (22) the undelayed measurement matrix (6) and the measurement noise covariance (15) are

$$
\mathbf{H}(\theta(k), \omega(k))=\left[\gamma \cos (\theta(k)) \quad \rho \eta \exp \left(\eta\left(\omega(k)-\omega_{0}\right)\right)\right]
$$

and

$$
\mathbf{R}=\sigma_{v}^{2}
$$

The augmented system then follow from equations (9), (10), (12), (13), (16)-(18), (20), and (21).
Table 1. Compression pump Monte-Carlo simulation parameter values.

$$
\begin{array}{lll}
\mu=0.6 \mathrm{~s}^{-1} & J=10 \mathrm{kgm}^{-2} & f_{V_{1}}=1 \mathrm{~Hz} \\
\nu=6 \mathrm{rads}^{-2} & \gamma=10^{4} \mathrm{~Pa} & \sigma_{w}=1 \mathrm{~V} \\
\eta=1 \mathrm{rad}^{-1} \mathrm{~s} & \rho=10^{5} \mathrm{~Pa} & \sigma_{v}=10^{3} \mathrm{~Pa} \\
\omega_{0}=30 \mathrm{rads}^{-1} & V_{0}=25 \mathrm{~V} & d t=0.01 \mathrm{~s} \\
R=100 \Omega & V_{1}=5 \mathrm{~V} & d t_{y}=0.1 \mathrm{~s}
\end{array}
$$

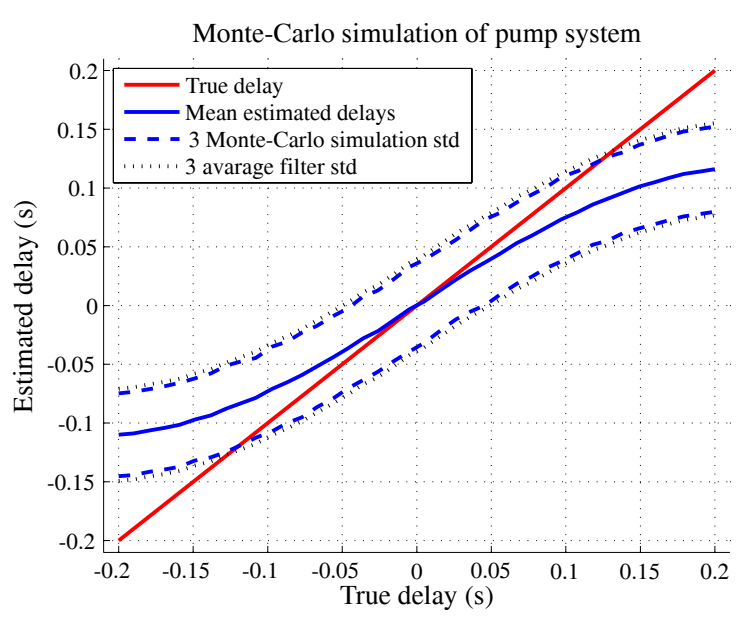

Figure 1. Mean and 3 standard deviations (std) around mean of estimated time-delay based on 1000 Monte-Carlo runs. The agreement between the true delay and the estimated delay is good for small delays while systematic errors appear for larger values. Compare the magnitude of the delay with the sampling period of $0.01 \mathrm{~s}$.

Applying a constant plus a sinusoid input to the system

$$
u(t)=V_{0}+V_{1} \sin \left(2 \pi f_{V_{1}} t\right)
$$

estimating the system state and the measurement time delay by the filtering equations given in Section 5, and running a Monte-Carlo simulation with 1000 runs with the parameter values of Table 1 , gives the mean estimated timedelays and variance after $10 \mathrm{~s}$ as a function of the true delays shown in Figure 1. At this point in time the filter mean has converged. The average filter standard deviation is the square root of the mean third diagonal element of the $\mathbf{P}_{k}$ matrix, over all Monte-Carlo runs, while the simulation standard deviation is the square root of the mean variance of the delay estimate, over all Monte-Carlo runs. The standard deviations are seen to agree well. Further, as expected the delay estimate behaves well at small values but has systematic errors at larger values since the linearization of the measurement in (4) becomes less accurate. As noted in Section 5, a way to remove the systematic error is to change the linearization point with the estimated delay. 


\section{CONCLUSION}

In this article we have suggested a novel approach to joint state and measurement time-delay estimation of a nonlinear state space system. The approach is based on a linearization in time of the measurement equation which leads to an easily evaluable EKF for an extended system augmented with the time-delay. The feasibility of the approach has been shown by an example and simulations. From the simulations the linearization in time is seen to give good estimation performance for small time-delays.

\section{REFERENCES}

[1] S. J. Julier and J. K. Uhlmann, "Fusion of time delayed measurements with uncertain time delays," in Proc. ACC'05, vol. 6, pp. 4028-4033, Portland, OR, USA, 8-10 June 2005.

[2] M. Choi, J. Choi, J. Park, and W. K. Chung, "State estimation with delayed measurements considering uncertainty of time delay," in Proc. ICRA2009, pp. 39873992, Kobe, Japan, 12-17 May 2009.

[3] J.-O. Nilsson and P. Händel, "Synchronization and temporal ordering of asynchronous sensor measurements of a multi-sensor navigation system," in Proc. PLANS2010, Indian Wells, CA, USA, 4-6 May 2010.

[4] A. R. Ferreira and J. M. Fernandes, "A survey on time delay system estimation," in Proc. ECC'97, Brussels, Belgium, 1-4 July 1997.

[5] S. Björklund, "A survey and comparison of time-delay estimation methods in linear systems," Licentiate thesis, Linköping University, 2003.

[6] J. Fox, "Synchronization of input and output measurements using a Kalman filter," in Proc. ICMIC2006, pp. 429-434, Lanzarote, Spain, 6-8 February 2006.

[7] I. Skog and P. Händel, "Time synchronization errors in GPS-aided inertial navigation systems," IEEE Trans. on Intell. Transp. Syst., 2010. (In revision).

[8] D. Simon, Optimal State Estimation: Kalman, H-inf, and Nonlinear Approaches. Wiley-Interscience, 2006. 